\title{
Le symbolisme de la mer dans Les Plages d'Agnès Varda au miroir de la littérature
}

\author{
Corbí-Sáez, María Isabel
}

Universidad de Alicante, maribel.corbi@ua.es

\begin{abstract}
Resumen
La cinescritura de Agnès Varda ha transgredido numerosas barreras y abierto nuevos caminos en el séptimo arte. Les plages (2008) constituye un ejemplo claro de relato de arte y de ensayo, complejo, creado como una especie de collage, con efecto de caleidoscopio, en el que la cineasta ofrece une juego narrativo fílmico prolijo que seduce y atrapa al espectador lector cómplice. Este filme presenta un interés especial dado que si, efectivamente, hace un balance de una vida dedicada al cine y a las artes, si constituye asimismo de forma evidente una celebración del séptimo arte y un homenaje a numerosos amigos cineastas, ilustra también hasta qué punto el cine no puede concebirse sin las interinfluencias entre las artes. Nuestro análisis del simbolismo del mar descansa precisamente sobre un estudio del juego intertextual que Les plages establecen con la literatura.
\end{abstract}

Palabras clave : VardaФ; Les plages (2008); Mar ; simbolismo ; Intertextualidad ; Literatura ; Paul Valéry.

\begin{abstract}
Résumé
La cinécriture d'Agnès Varda a transgressé bien des barrières et ouvert de nouveaux chemins dans le septième art. Les plages (2008) constitue bien un exemple de récit d'art et d'essai, complexe, créé comme une espèce de collage, à effet de kaleidoscope, où la réalisatrice offre un jeu narratif filmique prolixe qui séduit et attrape le spectateur-lecteur complice. Ce film s'avère d'un intérêt spécial car si, effectivement, il fait le bilan de toute une vie dédiée au cinéma et aux arts, s'il constitue en toute évidence une célébration du septième art et un hommage à de nombreux amis cinéastes, il illustre jusqu'à quel point le cinéma ne peut être conçu sans les interinfluences entre les arts. Notre analyse du symbolisme de la mer repose précisément sur une étude du jeu intertextuel que Les plages établissent avec la littérature.
\end{abstract}

Mots-clés : A. Varda ; Les plages (2008) ; Mer ; Symbolisme ; Intertextualité ; Littérature ; Paul Valéry.

\begin{abstract}
Agnès Varda's cinécriture has transgressed many frontiers and has opened new paths in the seventh art. Les plages (2008) appears to be a very valid example of an art and essay cinema, complex, created as a kind of collage, with kaleidoscope effects, where this woman filmaker offers a prolix film narrative play which seduces the complicit spectator reader. This film presents a special interest in so far it constitutes not only a balance of a whole life devoted to the cinema and the arts, a clear celebration of the seventh art, an hommage to numerous Agnès Varda's film directors friends, but it also gives the measure to what extent the cinema must be considered taking into account the interrelations between arts. Our study on the sea symbolism bases precisely on the intertextuel play that Les plages establish with literature.
\end{abstract}

Keywords : A. Varda ; Les plages (2008); Sea ; Symbolism ; Intertextuality ; Literature ; Paul Valéry. 
Il roule par la brume, ancien et traverse

Ta native agonie ainsi qu'un glaive sûr ;

Où fuir dans la révolte inutile et perverse ?

Je suis hanté. L’Azur ! l’Azur ! l’Azur ! l’Azur !

(Stéphane Mallarmé)

Un beau vers renaît, indéfiniment de ses cendres, il redevient comme l'effet de son effet, cause harmonique de soi-même

(Paul Valéry).

Au premier abord Les plages semble constituer le voyage « en arrière » d'une vie, une suite rétrospective de portraits et d'autoportraits, une « rêverie autobiographique » d'une réalisatrice constamment au-devant d'une avant-garde. Si le jeu de miroirs dans lesquels se projette la protagoniste dans le générique ${ }^{1}$ pourrait faire penser que le texte filmique va tourner autour d'elle, le spectateur complice, cependant, comprend très vite dès les premières minutes que cela ne peut être qu'une illusion. Agnès Varda nous offre d'ailleurs à l'aide de divers cadrages des images d'elle-même voilées, incomplètes ou déformées ${ }^{2}$, ou encore exprime des paroles qui invitent le spectateur lecteur à soupçonner que ce film est beaucoup plus qu'un récit de vie, qu'il va s'agir d'une aventure textuelle dépassant les frontières entre les arts. En effet, s'il est un aspect incontournable de cette œuvre filmique, c'est bien qu'elle met en évidence le caractère polyvalent de son auteure, sa formation plurielle très solide, son dévouement à diverses disciplines artistiques, et sa passion pour la littérature. L'analyse du symbolisme de la mer dans cette œuvre ne peut s'entrevoir et s'apprécier en tournant le dos à certains textes artistiques, en général, et littéraires, en particulier ; des textes qui ont marqué l’imaginaire de la cinéaste comme nous aurons l'occasion de le développer.

Dans Les Plages, Agnès Varda rend compte de sa formation depuis les temps de l'école du Louvre, de ses lectures, des moments, des rencontres et événements qui ont marqué sa trajectoire professionnelle, d’abord comme photographe puis comme réalisatrice et finalement comme artiste intégrale. Elle revisite ses textes filmiques, ceux de ses amis cinéastes de la Nouvelle Vague ou encore ceux de la Rive gauche, les commente, mentionne certains amis artistes qui ont contribué à l'inspirer et à enrichir son œuvre, élabore des réécritures filmiques d'autres œuvres d'art, insère des séquences de certaines de ses installations, etc. Cette œuvre - un « postscriptum » selon Maxime Scheinfeigel (2009: 197) - dévoile au rythme des caprices de la mémoire de la réalisatrice, des instantanées de sa vie, de celles de ses amis, ses émotions et les passions vécues... Pourtant nous considérons que dans ce richissime longmétrage - un chef d'œuvre pour nombre de critiques - le caractère testimonial d'un récit de vie n'est certes que l'aspect le plus superficiel et correspondrait à un premier niveau de lecture.

Ses films précédents l’attestent. Sa cinécriture se veut en rupture constante avec un cinéma commercial, en refusant la tentation des récits traditionnels élégants - eux, fondés sur cette vocation séculaire mimétique et référentielle -, en pratiquant cette mixité des genres recherchée par un cinéma d'avant-garde, en fondant l'imaginaire et le réel, en exploitant les jeux du champ et du hors champ aussi bien visuel que sonore, en jouant sur le potentiel novateur de certains codes spécifiques, parmi beaucoup d'autres éléments définitoires ${ }^{3}$. Or s'il est un aspect incontournable dans les

\footnotetext{
${ }^{1}$ Cf. séquence (00: 00- 05:39). Le film commence par un jeu de miroirs réels et tangibles dans lesquels l’image complète d’Agnès Varda se dérobe constamment, cédant le protagonisme à la mise en scène du tournage proprement dit sur une plage avec les membres de l'équipe, ou encore à la mer lors de cadrages très significatifs. Cette réflexion de son visage ou d'une partie de son corps sur un miroir que la caméra filme constitue des jeux qui reviennent souvent dans ses films et acquièrent différentes portées et nuances. À commencer par la captation de l' « instant prégnant » à l'instar du peintre ou du photographe dans la technique du portrait ou de l'autoportrait, puis l'exploitation du miroir pour marquer la dualité de l'être et la vanité de toute tentative de saisie identitaire, ou encore le recours à l'autoportrait cinématographique à l'aide des miroirs cadrés par le viseur de la caméra pour marquer certains jeux énonciatifs (la réalisatrice participant comme personnage du récit), parmi beaucoup d’autres finalités. Pour ce qui est du dispositif du miroir et de la réflexion partielle de la réalisatrice voir l’intéressante étude d’Esteve Riambau, « La caméra et le miroir » (2009 : 135-143).

${ }^{2}$ Cf. l'utilisation d'un miroir abîmé par le passage du temps où la réflexion de l'image de la réalisatrice est complètement brouillée et défromée par les tâches noires : (cf. par exemple, plan 01:54).

${ }^{3}$ Il est fort connu qu'Agnès Varda n’apprécie pas beaucoup qu’on la définisse comme « la grand-mère de la Nouvelle Vague » puisqu’elle a toujours revendiqué un chemin personnel dans le cinéma d'art et d'essai qu'elle a pratiqué tout au long de sa profession. Pourtant, il faut situer ses débuts aux côtés de réalisateurs du cercle de la Rive Gauche (Alain Resnais, par exemple) et autour de ses amis les jeunes turcs (les jeunes critiques des Cahiers du cinéma) qui donneront lieu à la Nouvelle Vague. Si, effectivement, chaque groupe revendiquait différents types d’innovations, la conception du septième art qu'ils ont défendue s’érige contre un cinéma commercial, de grands budgets et de vaste production, tourné dans d’immenses studios. Ce nouveau cinéma de la fin des années cinquante qu'ils revendiquent sort dans la rue, caméra à l'épaule, ose affronter les difficultés du traitement de la lumière naturelle, cherche à présenter le vécu des personnages, leurs processus introspectif, se plonge dans le monde des perceptions, des sensations et du souvenir, et se propose de retransmettre des émotions et impressions qui dérivent de l'expérience individuelle. Ce ne sont plus
} 
films de Varda, et très particulièrement dans le cas de celui qui occupe notre analyse, c'est bien celui d'exploiter un langage filmique qui a conscience de soi, qui dans ce retour sur soi qu’il effectue, dévoile les mécanismes de sa production, de sa création, de la réception et surtout son caractère textuel et les jeux intertextuels qui s’y déploient. Cette réflexion de soi si pratiquée dans le cinéma d’avant-garde - un élément définitoire de celui-ci, d'ailleurs donnant lieu au discours métacinématographique qui devient le miroir où se reflète toute son œuvre ${ }^{4}$.

Grâce aux premières minutes du film et au titre qui l'accompagne, les spectateurs avisés prennent conscience du jeu établi dès le paratexte et du pacte de lecture. Les plages présentent des espaces maritimes très importants dans la vie de la réalisatrice. Pourtant à un deuxième degré, elles sont d’une toute autre nature, ce sont celles qui reçoivent les « vagues textuelles » qui entrelacent le récit. Et, puisqu’il en va de plages, de vagues, il en va également de «mer ». Une mer dont le caractère symbolique ne peut échapper au spectateur complice qui partage un certain bagage culturel et littéraire avec la réalisatrice. Ce n’est pas un hasard que vers la moitié du film un fragment du Cimetière marin de Paul Valéry soit récité par cœur par Andrée Villard, malade d’Alzheimer, - veuve de Jean Villard, le directeur du Théâtre National Populaire, un grand ami de la réalisatrice. La récitation de ces quelques vers et, de ce fait, la présence du célèbre poète de Sète jouent à notre avis un rôle fondamental dans cette œuvre filmique, puisque comme nous le verrons dans notre analyse, ce sont les thèses de Paul Valéry sur la création et l'art qui sont interpellées. Cette rêverie « autobiographique » peu conventionnelle, cette aventure textuelle que constitue Les Plages tisse un discours sur la question de la présence de l' « auteur/créateur » dans l’œuvre d'art ${ }^{5}$.

Du retour et la visite à la maison de naissance de Bruxelles occupée depuis des décennies par de nouveaux propriétaires, le bond en arrière à l'hommage reçu pour La Pointe courte (1955) qu'on lui rendit dans la capitale et le saut à la plage de Sète nous semblent très significatifs. " Je retourne à cette plage en arrière comme dans tout ce film » affirme la narratrice. Sète en fait a constitué le point de départ de nombreuses expériences qui auront une répercussion sur tout son devenir aussi bien personnel que professionnel. La séquence de la plage de Sète, une plage et une ville à laquelle elle reviendra souvent, est dans ce sens très révélatrice. C’est ici qu’elle a décidé de prendre son indépendance, qu'elle a perdu l'innocence nous dit-elle, mais c’est aussi ici qu'elle a pris conscience que le cinéma doit rompre avec sa vocation de représentation (ce qu'elle va illustrer en rappelant et reprenant des séquences du tournage de la Pointe courte). Si la séquence des trapézistes sur la plage est importante parce qu'elle fait allusion aux origines du cinéma comme spectacle, elle l'est d'autant plus à notre avis car elle comporte également une clé de lecture de l'œuvre. Les rideaux de filets ${ }^{6}$ ou les filets ${ }^{7}$ qui reviennent souvent agissent comme des métaphores du caractère textuel de l'œuvre d'art. Nous l'annoncions précédemment « Les plages » d'emblée ont pour référents immédiats les espaces géographiques maritimes réels qui ont marqué la vie de la réalisatrice, et au deuxième degré « ces plages » renvoient à l'ensemble des textes artistiques, dont les filmiques parmi tant d'autres. Ce parcours qu'elle fait en arrière, n'est qu'un subtil prétexte pour revisiter toute son œuvre au gré de sa mémoire et surtout au gré des défis et « acrobaties » de la création considérée comme un jeu. Ce texte qui défile sous nos yeux est tissé, ne l’oublions pas, à partir de nombreux textes précédents, les siens et ceux des autres. Arrivé à ce point, le spectateur-lecteur ne peut s'empêcher de se souvenir du générique avec le gros plan du miroir qui ne reflétait que la mer et où la voix en off disait « situé de cette façon la force du vent du nord le

des histoires compactes, faciles à suivre avec leur début et leur fin bien précises, racontées avec des dialogues et des images concaténées selon un ordre chronologique qui intéressent ces jeunes réalisateurs assoiffés de renouveau. Ceux-ci vont pratiquer avant tout des récits qui entrelacent plusieurs fils narratifs, plusieurs genres, qui jouent sur l'ambigüité, sur l'opacité, sur la contradiction, sur les silences et les ellipses, la discontinuité et la fragmentation, l'esthétique formelle occupant une place privilégiée. Le montage de la bande image et de celle du son répond également à cette volonté de rupture. Toute une conception du septième art qui va exiger chez le spectateur une complicité incontournable.

${ }^{4}$ Cf. à cet égard notre étude « Reflexividad en Les plages de Agnès Varda. Un viaje por los textos como espejo de su cinécriture » (Corbí-Sáez 2012 : 109-129).

${ }^{5}$ L'abordage du jeu intertextuel d'une œuvre s'avère une vaste et ambitieuse entreprise, d'ailleurs pleine de risques dans le dépistage des textes. Ici, nous nous limitons à voir les enjeux des références à certains textes littéraires dont celui de Paul Valéry en rapport à cette présence du créateur dans l'œuvre d'art. Les limites d'extension des articles nous imposent un choix. Nous réservons pour un futur proche une version plus ample de cette question.

${ }^{6}$ Profitant cette séquence qui recrée un spectacle de cirque sur le sable, la voix off commente son passé et sa découverte de la sexualité. Le cadrage du plan d’ensemble sur deux êtres entrelacés en deuxième position et au devant deux femmes acrobates qui tirent et ferment à nouveau les rideaux de filets est à notre avis essentiel quant à la définition du septième art que poursuit Agnès Varda et dont elle rend compte dans Les plages. Ici, la réalisatrice suggère qu'elle a abandonné avec la perte de l'innocence, cette volonté première de représentation et de vérisme de l’art cinématographique, et que le seul spectacle qu’elle recherche est celui que les sauts et les péripéties de la création lui offrent, c’est-à-dire le spectacle de sa cinécriture. (Cf. séquence 28:02-29:23).

${ }^{7}$ Voir importance du plan d'ensemble de la jeune femme assise au bord de la jetée réparant un filet. Ceci acquiert à notre avis une valeur symbolique et agit comme une mise en abyme de la création artistique renvoyant au caractère textuel de cette dernière. (Cf. plan, par exemple, 36:55). 
maintient stable », en affirmant plus loin « Les plages n’ont pas d’âge ». Et nous ajoutons : elles sont éternelles comme les œuvres d’art, la force du vent de l’inspiration et, surtout, celui de la création aide à les perpétuer.

Les plages constitue un texte richissime quant aux allusions et clins d'œil aux œuvres littéraires. À notre avis, en arrière-plan nous pouvons trouver des allusions, par exemple, à celles qui tissent des quêtes identitaires tel que le Portrait du jeune adolescent parmi d'autres, telle que l'œuvre proustienne avec le traitement de la mémoire et la quête du moi. La narratrice commente souvent quelles ont été les œuvres importantes pour sa formation, surtout comme embrayeurs de la création de ses films, l’intertextualité au-delà des frontières entre les arts jouant donc un rôle principal dans la création. Le roman de Faulkner Les palmiers sauvages qui, avoue-t-elle, lui inspira La pointe courte, Sans toit ni loi qui, nous dit-elle, fut un hommage à Nathalie Sarraute ${ }^{8}$... Les fragments insérés de Cléo de 5 à 7 ne peuvent ne pas faire penser aux personnages exploités par les nouveaux romanciers et, en particulier, aux personnages féminins de Marguerite Duras.

Par ailleurs, la réalisatrice dévoile dans ce dernier longmétrage son admiration envers les écrivains et poètes de la modernité et avoue qu'elle leur a cédé dans ses œuvres un espace notoire. Dans Les Plages, outre ceux que Varda énumère tels que Baudelaire ${ }^{9}$, Apollinaire, Rilke, Prévert, Brassens..., sans oublier Paul Valéry sur lequel nous reviendrons postérieurement, la mention aux poètes et écrivains surréalistes en est également incontournable ${ }^{10}$. Avec la référence à L'amour fou d'André Breton, le spectateur lecteur peut retrouver à son esprit l'idéaire artistique du groupe de jeunes qui rompirent avec les paramètres d'un art conventionnel, qui inspirèrent et nourrirent des générations d'artistes à venir. Les séquences liées à ce mouvement sont nombreuses et nous semblent très révélatrices quant à certains aspects. Varda avoue avoir été subjuguée par l'avant-garde surréaliste dans sa jeunesse. Nous retenons le caractère pluridisciplinaire et intégrateur du mouvement que la réalisatrice dans ses lectures et études de Lettres à très bien saisi et qui constitue sans aucun doute l'un des fondements premier de sa conception de l'art. Ainsi, la mention par la voix en off du « cadavre exquis » agit comme une espèce de mise en abyme d'un type d'écriture artistique (collective et séquentielle, technique du collage...), des traits qui nous rappellent certains aspects de ses textes filmiques depuis ses débuts jusqu’à celui que nous avons sous les yeux.

Sa connaissance de la littérature est beaucoup plus ample, ayant passé un « Bacho de Lettres », il n’est pas surprenant d'entendre dire à la narratrice en voix en off « [...] je connais mes classiques ». « [...] Connais tes classiques » affirmet-elle ensuite en interpelant la complicité de son spectateur lecteur car bien souvent les cadrages comportent des références et allusions à ces textes au-delà des frontières entre les arts. Un exemple intéressant est celui d'un plan séquence de l'homme et de l'enfant nus regardant la mer et tournant le dos à la caméra, d'abord avec une image numérique passant progressivement à l'image conventionnelle dans un plan petit à petit rapproché. Une composition et un cadrage qui interpellent de nombreuses œuvres de disciplines différentes inspirées du texte fondateur des odyssées, y compris celles de Varda telle que Nausicaa (1970), Ulysse (1984) ${ }^{11}$. Ce n’est pas un hasard que dans cette séquence, la voix en off de la narratrice commente : «[...] J'aime les enfants qui s'appellent Ulysse, les hommes qui regardent la mer s'appellent tous Ulysse [...] ce sont des Ulysse que ne veulent pas rentrer à la maison » ${ }^{12}$. Allusions aux voyages initiatiques, aux quêtes identitaires pour nous dire que, pour elle, ce sont surtout les voyages « en arrière » à travers les

\footnotetext{
${ }^{8}$ Dans Les plages le gros plan dédié à l'écrivaine est significatif. Rappelons également le plan de Nathalie Sarraute dans le générique de Sans toit ni loi. Nous annonçons pour un avenir proche une étude sur la portée de cette inspiration et surtout sur les tropismes version cinématographique.

${ }^{9}$ De fait les spectateurs lecteurs connaisseurs de l'auteur de Les fleurs du mal ne peuvent s'empêcher de penser à la célèbre strophe de « L’Homme et la mer » : « Homme libre, toujours tu chériras la mer !/ La mer est ton miroir; tu contemples ton âme/Dans le déroulement infini de sa lame/Et ton esprit n’est pas un gouffre moins amer [...] ». L'écriture poétique pour Baudelaire est le seul miroir où se reflète son moi. Évidemment, il faut voir cet espace du jeu de réflexion et de distance que Varda établit avec l'intertexte du poème de Baudelaire, aspect que nous développons par la suite.

${ }^{10}$ Tel qu'indiqué dans notre introduction, la longueur limite de l'article ne nous permet pas d'aborder les innombrables jeux intertextuels avec d'autres disciplines artistiques tel que la peinture, par exemple. Nous ne pouvons nous empêcher de signaler l'importance que le peintre surréaliste Magritte acquiert. Nous trouvons dans ce récit filmique une réécriture de son tableau Les amants et dans le discours sur la question de l'autoportrait de l'artiste, le spectateur avisé peut également déceler des réminiscences très intéressantes. Les plages interpellent souvent les autoportraits de Magritte, l'autoportrait de dos qui offre la ligne de la silhouette du peintre et l'espace de la tête étant seul rempli de ciel/mer. La tête de l'artiste étant l'espace où transite la « mer » - « cet Azur » selon Stéphane Mallarmé... Nous reprendrons cette question
} dans un prochain article.

${ }^{11}$ La filmographie d'Agnès Varda présente de nombreux titres qui abordent le voyage comme quête identitaire et le voyage à travers les textes.

${ }^{12}$ Cf. séquence (28:09-28:26). 
textes, les siens et ceux des autres qui l'intéressent comme artiste ${ }^{13}$. Pour une «rêverie autobiographique » cela peut sembler étrange. Le spectateur avisé prend cependant bien conscience que l’enjeu du film se situe ailleurs.

Si, tel que nous l'avons commenté antérieurement, Varda avoue à plusieurs reprises qu'elle n'aime pas parler d'elle, qu'elle ne le fait que de façon voilée, la séquence de la visite de sa maison de naissance occupée par d’autres propriétaires depuis des décennies apporte une clé de lecture supplémentaire. Revenir à ses origines pour se définir et construire son identité n’a pas beaucoup de sens pour la réalisatrice, puisqu’elle « n’a pas la nostalgie de son enfance » et n'a «[...] ni souvenirs de peines ou de larmes », vouloir retrouver ses racines non plus puisque toute tentative de définition d'une identité personnelle repose sur une construction et avec elle une part d'invention. De fait, avoue-t-elle, que son prénom d'origine fut celui d' « Arlette » qu'elle changea pour celui d' « Agnès ». « Tenter de reconstruire des scènes de son enfance sont en fait un contresens » dit la narratrice. Donc aucun intérêt à vouloir récupérer par la représentation cette étape. Les Plages, ainsi, dès les premières minutes dévoilent que Varda va s'éloigner en tout et pour tout du récit autobiographique et de l'autoportrait conventionnel. Ce n'est pas le moi biographique qui l'intéresse, elle préfère plutôt l'artistique. La mention à Gaston Bachelard est dans ce sens révélatrice. Se souvenant des cours de la Sorbonne et de l'explication reçue du mythe de Jonas, Varda reconstruit à la façon d'une installation sur la plage une immense baleine, se présente comme un personnage dans un plan rapproché à l'intérieur de sa création, avoue à la caméra que c'est à l'intérieur du ventre de l'animal qu'elle se sent protégée et qu’à l'instar de Jonas elle se résiste à en sortir. C’est dans le « ventre » de l'art qu'elle veut rester ${ }^{14}$.

Nous l'annonçons précédemment, Paul Valéry occupe à notre avis une place de prime importance dans ce discours sur la création et la question de la présence de l'auteur/créateur dans l'œuvre d'art. Un texte filmique qui aurait pour but d'offrir une suite d'autoportraits et de revisiter les œuvres et la trajectoire professionnelle de son réalisateur comporterait en principe selon la convention un certain égotisme, un égotisme absent tout du moins à la façon traditionnelle dans cette aventure textuelle que constitue les Plages de Varda. Le fragment du Cimetière marin récité est à notre avis très éloquent pour deux raisons. D’une part, c'est une malade d'Alzheimer qui fait une récitation par cœur des vers. Aspect, certes, non négligeable quant à sa portée. Il est fort connu que cette maladie entraîne une dégénération de la mémoire à tel point que les individus perdent la conscience de leur identité et de celle des autres. Andrée Vilar récite par cœur ces vers sans avoir conscience de leur auteur.

Ce toit tranquille, où marchent des colombes,

Entre les pins palpite, entre les tombes,

Midi le juste y compose de feux

La mer, la mer, toujours recommencée ! (Cf. séquence 27:06-28:02).

D’autre part, en écoutant ce fragment, les spectateurs lecteurs avisés et connaisseurs de l'œuvre poétique de Valéry ne peuvent manquer l'allusion à la réflexion sur la condition humaine et la finitude, et à l'art comme absolu. De fait, le célèbre vers «La mer, la mer toujours recommencée » interpelle d'innombrables textes poétiques qui ont exploité la mer comme symbole de l'art qui, lui, est éternel. Une mer qui au rythme des flots déversent à l'infini sur les plages les œuvres d'art.

Cependant, pour apprécier davantage la portée de cette séquence en rapport au symbolisme de la mer, les spectateurs lecteurs doivent tenir compte du commentaire de la voix off de la narratrice. Ainsi affirme-t-elle « La poésie est ce qui reste quand la mémoire s’en va ». Si, effectivement, il nous semble que par " poésie » nous devons également concevoir amplement la création artistique tel que Cocteau l’a définie (poésie de poésie, poésie de roman, de cinéma, poésie de peinture...), ces paroles nous renvoient à la question de la création artistique et du but de celle-ci. La mention des vers de Paul Valéry constitue une mise en abyme de l’un des fils thématiques du texte filmique de l'art comme témoin du temps et de son caractère textuel dans lequel le créateur - la personne biographique dotée d'une psychologie, de sentiments, etc. - n'a aucune place. Nous l'avons signalé, Andrée Vilar remémore les vers sans savoir à qui ils

\footnotetext{
${ }^{13}$ Le plan qui culmine la séquence de l'homme et l'enfant nus regardant la mer est dans ce sens très révélateur, la réalisatrice apparaît tournant le dos à la caméra et s'approchant des personnages pour les couvrir.

${ }^{14}$ Cf. séquence (35:29-36:09).
} 
correspondent, donc ceci renvoie d'emblée à ce manque d'intérêt et de pertinence de la présence de l'auteur dans l'œuvre d'art. Il s’agit surtout de l'«état poétique » que le "poète » a été capable de retransmettre à ses lecteurs à travers le langage poétique et que les lecteurs actualisent constamment.

Nous rappelons que l'état poétique pour Paul Valéry n’est pas celui qui résulte de l'expérience sensuelle (à prendre au sens littéral, propre aux sens) directe et immédiate de l'artiste à la façon des romantiques voire même encore des symbolistes ${ }^{15}$ qui, dans leur besoin d'exprimer leur moi intime et leur subjectivité, eurent recours à un langage fondé sur des images, des symboles et des formes particulières, un langage très alambiqué. L'état poétique, par contre, est le résultat d'un processus de transformation rationnelle de premiers états mentaux substantiellement irrationnels. Pour l'auteur du célèbre Cimetière marin la création doit compter fondamentalement sur l'activation consciente de l'intellect, et à partir d'un effort d'abstraction et d'épuration pouvoir choisir les formes et les structures linguistiques capables de recréer dans le versant lecteur cet état poétique.

C’est dire que cet état de poésie est parfaitement irrégulier, inconstant, involontaire, et que nous le perdons comme nous l'avons obtenu par accident. Mais cet état ne suffit pas pour faire un poète, pas plus qu'il ne suffit de voir un trésor en rêve pour le retrouver, au réveil, étincelant au pied de son lit.

Un poète - ne soyez pas choqué de mon propos - n’a pas pour fonction de ressentir l'état poétique ceci est une affaire privée. Il a pour fonction de le créer chez les autres (Valéry, 1957 : 1321).

Conscient de sa fonction, conscient de sa tâche et de son savoir-faire, le poète, voire l'artiste, doit être capable de détecter en soi-même cet état poétique et à l'aide du langage - dans le cas de Valéry, le poétique, dans le cas de Varda le filmique, doit construire lucidement, comme s’il s'agissait d'un labeur, de façon calculée le poème capable de déchaîner dans le lecteur une expérience similaire basée sur une expérience mentale consciente et lucide, un lecteur qui selon Valéry est « le ressort de la puissance poétique ». Nous soutenons que la séquence qui récupère les vers de Paul Valéry est de prime importance car elle permet au spectateur lecteur avisé d’appréhender définitivement d’une façon beaucoup plus nuancée la portée du longmétrage vardien qui s'est proposé boucler toute une trajectoire professionnelle. Nous l'avons vu précédemment, son richissime texte filmique est tissé à l'aide de nombreux autres textes antérieurs, les siens et ceux de ses amis, et nous le rappelons, des textes qui relèvent de diverses disciplines artistiques. Dans toutes les séquences la voix de la narratrice - soit in soit off - narre, commente et explique les aspects liés aux œuvres d'art revisitées. Une voix douce et tendre qui prédispose le spectateur lecteur à récupérer cet état poétique expérimenté par chaque «plage » revisitée ainsi que par Les plages, des émotions senties par ses spectateurs lecteurs car, Varda, depuis ses débuts a bien cherché dans son langage filmique l'émotion : J'ai travaillé si dur à chercher depuis la Pointe courte, quelque chose qui vienne de l'émotion, de l'émotion visuelle, de l'émotion sonore, en sentant et trouvant une forme, une forme qui tienne du cinéma et de rien d'autre [...] (Varda in Quart, 1986/1987 : 4, c'est notre traduction).

Les plages - film d'art et d'essai - que Varda a légué en fin de carrière à son public, qui tisse et combinent « des images qui l'ont habitée depuis longtemps » selon la réalisatrice, au rythme de la progression, d’un enchaînement et d’un entrelacement très calculé des séquences, nous l'annoncions dans les premières lignes de notre analyse, attrape le spectateur lecteur par cette émotion qui émane du langage filmique. Une émotion qui procède de la création artistique et de cet état poétique que ces vers de Paul Valéry nous ont rappelés. Pour le poète originaire de Sète, le poème :

[...] ne meurt pas pour avoir vécu : il est fait expressément pour renaître de ses cendres et redevenir indéfiniment ce qu'il vient d'être. La poésie se reconnaît à cette propriété qu'elle tend à se faire reproduire dans sa forme : elle nous excite à la reconstituer identiquement (Valéry, 1957 : 1331).

\footnotetext{
${ }^{15}$ Paul Valéry concède une importance incontournable à l'intellect dans l'acte créateur au détriment du sensible. Le poète de Sète dépasse les symbolistes rompant avec le recours abusif aux symboles, à la magie et à la sorcellerie évocatoire liée à la musique, renonçant à l'expression de l'intérieur du poète, de ses sentiments, de sa perception du monde et de la multiplicité des sens à travers le langage, les images et les formes. Pour l'auteur du Cimetière marin, les symbolistes certes ont fait de grands efforts pour frayer les chemins des innovations du langage poétique et de la poésie, des innovations annonciatrices de toute une poésie à venir dans les décennies suivantes. Pourtant chez certains poètes symbolistes l'œuvre est encore considérée comme le fruit d’une imagination et d'une sensibilité, de là l'espace encore dédié à la subjectivité. Un espace de subjectivité que Paul Valéry s'est proposé d'évincer en parlant de la « poésie pure ».
} 
[...] Un beau vers renaît, indéfiniment de ses cendres, il redevient comme l'effet de son effet, cause harmonique de soi-même (Valéry, 1957 : 1510).

Les plages dans leur tissage ont bien repris ces «beaux vers » qui renaissent indéfiniment de leurs cendres, Les plages viennent donc intégrer cette « Mer sans cesse recommencée ».

\section{Références bibliographiques}

Bluher, Dominique (2009). « À propos de quelques films et installations d’Agnès Varda », dans Fiant, Anthony ; Hamery, Roxane et Thouvenel, Éric (sous la direction de) Agnès Varda : le cinéma et au-delà. Rennes : Presses Universitaires de Rennes, 177- 188.

CORBI-SAEZ, Mª Isabel (2012). « Reflexividad en Les plages de Agnès Varda. Un viaje por los textos como espejo de su cinécriture », dans Bagué Luis (ed.), Un espejo en el camino, Madrid: Verbum, 109-129.

FEVRY, Sébastien (2000). La mise en abyme filmique. Liège : Editions du Céfal.

QUART, Barbara (1986/1987). « Agnès Varda : a conversation », dans Film Quarterly (n 2, 3-10).

Riambau, Esteve (2009). « La caméra et le miroir : portraits et autoportraits », dans Fiant, Anthony ; Hamery, Roxane et Thouvenel, Éric (sous la direction de), Agnès Varda : le cinéma et au-delà. Rennes : Presses Universitaires de Rennes, 135-143.

SCHEINFEIGEL, Maxime (2009). «Par-delà le cinéma, installer des images », dans Fiant, Anthony, Hamery, Roxane, Thouvenel, Éric (sous la direction de), Agnès Varda : le cinéma et au-delà. Rennes : Presses Universitaires de Rennes, 189-198.

VAlery, Paul (1980). « Mémoires du poète », dans Euvres. Paris : Gallimard, coll. Bibliothèque de la Pléiade, édition établie et annotée par Jean Hytier, vol I, 1980, 1447-1514.

VAlery, Paul (1980). "Théorie poétique et esthétique », en Euvres. Paris : Gallimard, coll. Bibliothèque de la Pléiade, édition établie et annotée par Jean Hytier, vol I, 1980, 1153-1418. 